\title{
A INFLUÊNCIA DA GEOMETRIA DO ESPARGIDOR NA INJEÇÃO E QUEIMA DE BAGAÇO DE CANA DE AÇÚCAR
}

\author{
J. H. SOSA ARNAO ${ }^{1}$, D. J. O. FERREIRA ${ }^{2}$, C. G. SANTOS ${ }^{1}$, J. E. ALAVAREZ- JÁCOBO ${ }^{1}$, L. P. \\ RANGEL $^{3}$ e S. W. PARK ${ }^{2}$ \\ ${ }^{1}$ Equipalcool Sistemas, Departamento de Pesquisa e Desenvolvimento \\ ${ }^{2}$ Universidade de São Paulo, Departamento de Engenharia Química \\ ${ }^{3}$ ESSS Engineering Simulation and Scientific Software \\ contato: ${ }^{1}$ juan@equipalcool.com.br, ${ }^{2}$ chaada@gmail.com, sonwpark@usp.br, \\ 3 leonardo@esss.com.br
}

\begin{abstract}
A Fluidodinâmica Computacional (Computational Fluid Dynamics, CFD) é utilizada aqui para modelo de combustão heterogênea de bagaço de cana-de-açúcar com espargidor (swirl burner). O objetivo do presente trabalho é comparar a injeção e queima de bagaço para um novo espargidor em relação ao modelo atual, já operante em uma caldeira de cogeração de biomassa. Este novo projeto de espargidor permite o controle da injeção das partículas através de regulagem das contribuições axial/tangencial do ar de arraste. $\mathrm{O}$ modelo CFD desenvolvido representa a turbulência pelo modelo RNG k- $\varepsilon$, a combustão dos voláteis pelo modelo EDM (Eddy Dissipation Model) com mecanismo composto de duas reações de combustão de $\mathrm{CH}_{4}$ e $\mathrm{CO}$, a radiação representada pelo método DTM considerando 16 direções e o arraste das partículas de bagaço no meio contínuo gasoso é representado pelo modelo Schiller-Naumann com os diâmetros sob análise granulométrica. Os resultados obtidos são favoráveis à adoção do design novo de espargidor, pois através do controle dos ares axial e tangencial é possível até mesmo reproduzir a operação do espargidor atual.
\end{abstract}

\section{INTRODUÇÃO}

A queima de combustíveis sólidos em equipamentos como caldeiras e câmaras de combustão é fortemente influenciada pela sua estratégia de alimentação, pois sua combustão é governada pela volatilização e a queima desses voláteis. Dentre as formas de inserção de sólidos em equipamentos de combustão confinada está o uso de espargidores cujo ar de arrasto possui uma componente axial, responsável pela penetração do mesmo no interior do equipamento, e outra tangencial, que promove maior grau de mistura e espalhamento do material. A queima de bagaço de cana por meio da injeção com espargidores com componentes axial e tangencial (rotação) melhora a estabilidade da queima, atenua possíveis oscilações e depósito de material não queimado nas superfícies da caldeira, melhora o controle da combustão e permite a queima de bagaço com maior grau de umidade (Dixon et al., 2005).

No presente trabalho são realizadas simulações CFD para comparar a queima de bagaço de cana 
promovida por dois modelos de espargidores, um chamado modelo atual, já em operação, e um outro novo design de modelo, ainda não instalado. Outro trabalho, de Ferreira et al.(2014), complementa a análise com o modelo de fornalha de caldeira de bagaço onde este swirl é utilizado. O espargidor de bagaço é constituído de duas tubulações concêntricas, uma interna em que escoam o ar axial e as partículas de bagaço e outra externa em que escoa sob rotação o ar tangencial. As vazões de ar axial, tangencial e as partículas de bagaço interagem entre si próximas à saída do espargidor, a poucos centímetros da entrada no equipamento. No espargidor atual, o ar tangencial é direcionado em um dos sentidos horário ou anti-horário por uma válvula localizada logo na entrada passando em seguida pela tubulação externa num escoamento rotacional anular. A geometria de toda a tubulação externa por onde escoa o ar tangencial é estática. No novo modelo de espargidor, o sentido de rotação é imposto por um conjunto de pás localizado no anel externo à tubulação pela qual passa o ar axial e as partículas de bagaço. A posição do conjunto de pás pode variar axialmente para trás e para frente sendo que na configuração para trás o espargidor se encontra no arranjo chamado aberto que permite que parte do ar tangencial escoe entre as pás e seja direcionado sob rotação e parte escoe diretamente axialmente para a saída do equipamento. No arranjo fechado, as pás estão posicionadas totalmente na frente, impondo que todo o ar tangencial saia do anel externo sob rotação. As Figuras 1 e 2 apresentam as principais características do espargidor antigo e dos arranjos aberto e fechado para o novo modelo de espargidor.

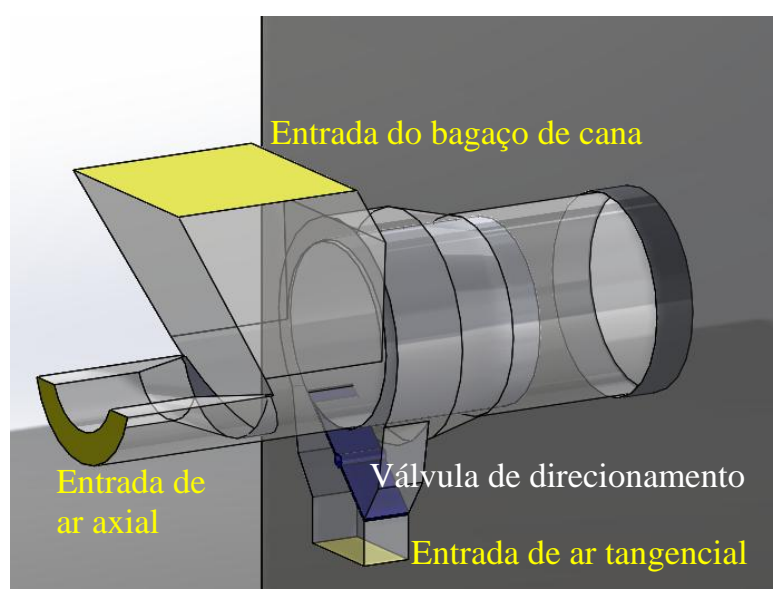

Figura 1: Principais carcterísticas do espargidor atual de cana.

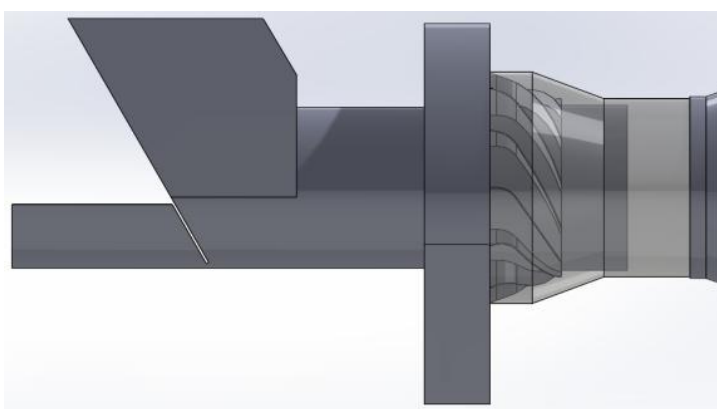

Aberto

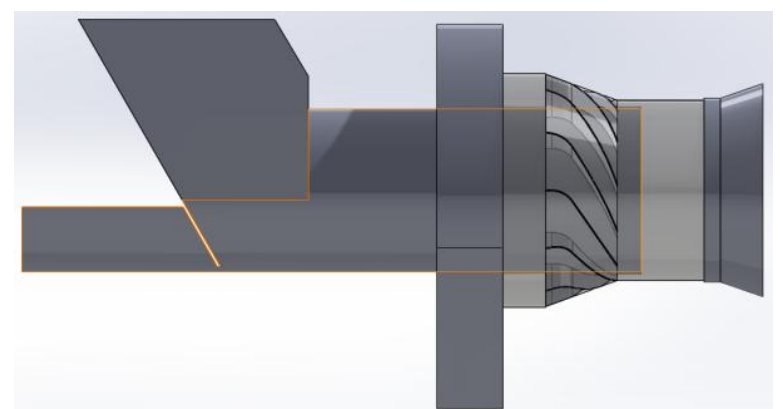

Fechado

Figura 2: Arranjos Aberto e Fechado para o espargidor novo de bagaço de cana 


\section{9 a 22 de outubro de 2014 \\ Florianópolis/SC}

\section{MÉTODOS}

Para o presente trabalho foram realizadas simulações com os modelos RANS de 2 equações k- $\varepsilon$ padrão e RNG k- $\varepsilon$ e os RANS de 6 equações BSL e SSG. De todos os modelos testados o que forneceu os resultados mais coerentes de acordo com a operação esperada para o equipamento e com

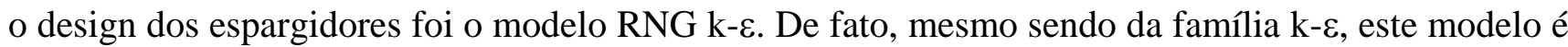
modificado e adequado a escoamentos sob rotação predominante (Versteeg e Malalasekera, 2007). A combustão em fase gasosa no interior da fornalha é representada pelo Modelo de Dissipação das Escalas Turbilhonares (EDM, Eddy Dissipation Model), pois é possível considerar que a operação da caldeira bagaço de cana favorece a disponibilidade suficientemente elevada de reagentes e temperaturas para que o processo de queima seja governado pela taxa de mistura dos gases reagentes (Turns, 2000), determinada pela escala de tempo turbulenta ( $\mathrm{k} / \varepsilon$ ou $1 / \omega)$. Para estimar as condições de contorno para a composição dos voláteis inseridos, são utilizadas análises imediata e elementar de amostras de bagaço de cana da região sudeste do Brasil, realizadas pela USP de Ribeirão Preto e pelo IPT-SP. As espécies consideradas são $\mathrm{CH}_{4}$ (representando todo o conjunto de hidrocarbonetos leves liberados na volatilização), $\mathrm{CO}, \mathrm{H}_{2} \mathrm{O}, \mathrm{CO}_{2}$ e $\mathrm{H}_{2} \mathrm{O} . \mathrm{O}$ mecanismo de reação é composto por duas reações químicas: $\mathrm{CH}_{4}+\mathrm{O}_{2} \rightarrow \mathrm{CO}+\mathrm{H}_{2} \mathrm{O}$ e $\mathrm{CO}+\mathrm{O}_{2} \rightarrow \mathrm{CO}_{2}$. A radiação é representada pelo DTM que considerada a emissão, reflexão e espalhamento da intensidade de radiação de forma isotrópica (Modest, 2003) para 16 direções igualmente espaçadas em todo o domínio computacional. Apesar do acoplamento em uma via ser adequado para representar a troca de quantidade de movimento e de massa entre as fases dispersa e contínua por causa da baixa concentração das partículas no domínio computacional, a troca térmica necessita de um acoplamento em duas vias. Se por um lado toda a fonte de combustível provém da volatilização e consumo do carbonizado das partículas de bagaço, por outro, para que tais reações heterogêneas ocorram, é necessário haver fluxos de calor da fase contínua gasosa para as partículas, ativando o processo. Portanto, há uma troca de influências em via dupla entre as fases consideradas. O modelo Schiller-Naumman para o arraste de particulados pode ser considerado adequado para a representação do bagaço de cana porque cada partícula de bagaço deve ser considerada rígida, sem deformação. A distribuição granulométrica das partículas é a utilizada em Sosa-Arnao (2008) para o bagaço de cana obtido após a moenda.

\section{SIMULAÇÕES}

Ao todo, foram realizadas simulações de escoamento isotérmico uniforme sem partículas, escoamento isotérmico bifásico com 10.000 partículas de bagaço e de combustão heterogênea de 10.000 partículas de bagaço para seis geometrias: espargidor antigo nos sentidos horário e antihorário e espargidor novo nos arranjos aberto e fechado para os sentidos horário e anti-horário. Nas simulações de escoamento isotérmico uniforme, apenas o escoamento promovido pelas geometrias foi avaliado. Nas simulações de escoamento isotérmico com a presença de partículas, se arraste também foi avaliado sob ação de campo gravitacional. Nas simulações de combustão heterogênea, a queima do bagaço é avaliada sob campo gravitacional e um escoamento ascendente de $2 \mathrm{~kg} / \mathrm{s}$ em volume de controle da simulação, ou farfield. A Figura 3 apresenta uma visão geral das condições de contorno adotadas tomando como exemplo uma das geometrias de espargidor novo. 


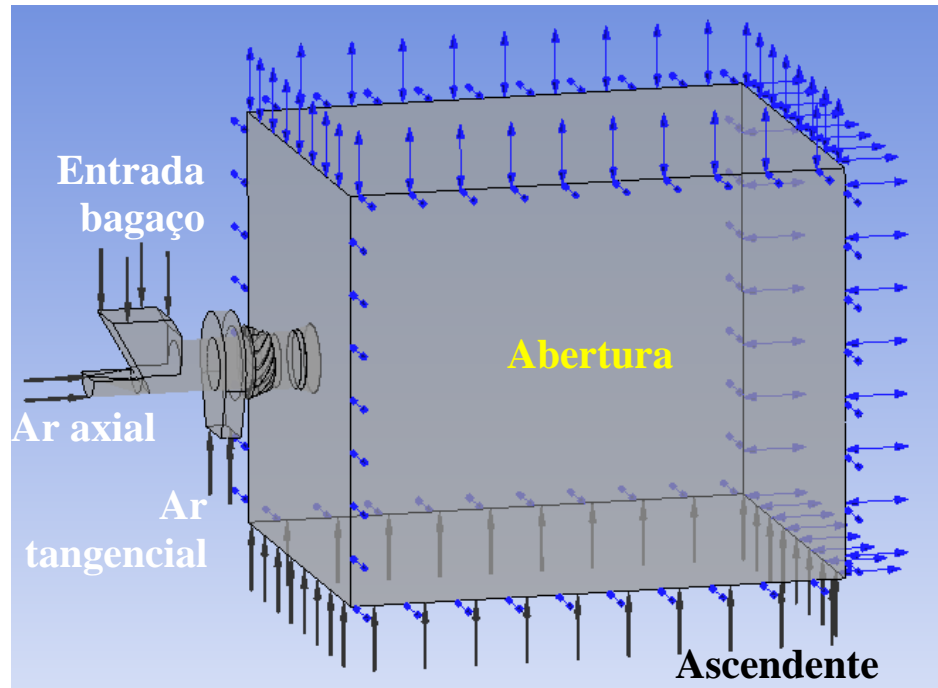

Figura 3: Condições de controno adotadas nas simulações dos espargidores

Com fins de simplicidade, no presente trabalho são abordados apenas os resultados das simulações de combustão heterogênea. A Tabela 1 apresenta as condições de contorno adotadas em todas as simulações.

Tabela 1 - Condições de contorno inseridas na simulação em regime permanente da caldeira de queima de bagaço de cana.

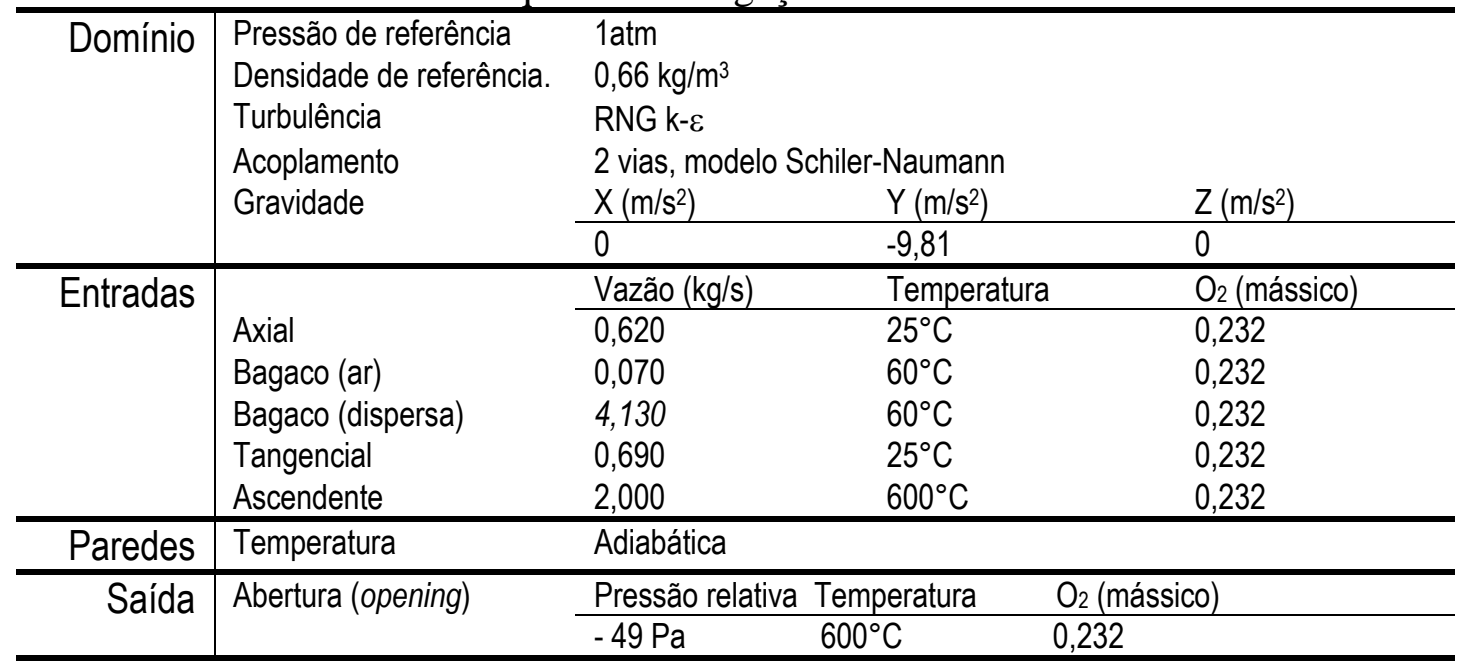


São simulados 6 casos:

Espargidor Atual :

1. Sentido Horário

2. Sentido Anti-horário

3. Sentido Horário Aberto

Espargidor Novo :

4. Sentido Horário Fechado

5. Sentido Anti-horário Aberto

6. Sentido Anti-horário Fechado

\section{RESULTADOS}

Com o objetivo de ser breve, em função da limitação de páginas do presente documento, esta sessão apresenta apenas os resultados obtidos para as simulações de combustão heterogênea do bagaço no arranjo anti-horário.

Os resultados obtidos para todas as simulações isotérmicas (homogêneas e bifásicas) apresentaram comportamentos espelhados para cada par de simulações com sentidos opostos e, portanto, no presente trabalho são exibidos os resultados para apenas um dos sentidos: o anti-horário. Como esperado, nas simulações isotérmicas, os resultados para o arranjo aberto do modelo novo apresentaram comportamento mais axial da fase contínua com pouco espalhamento das partículas e, consequentemente, maior penetração das mesmas no farfield. Já o arranjo fechado do espargidor novo apresentou maior espalhamento das partículas e melhor mistura. A geometria do espargidor atual apresentou um comportamento intermediário entre os encontrados para os arranjos aberto e fechado do modelo novo com espalhamento das partículas porém sua fase contínua apresentou menor rotação do que o esperado, em função do que foi projetado.

A Figura 4 apresenta perfis verticais iso-simétricos de velocidade para os casos simulados.

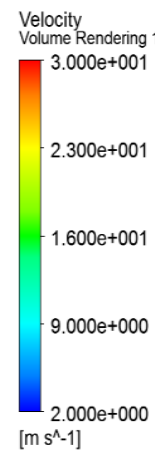

Atual

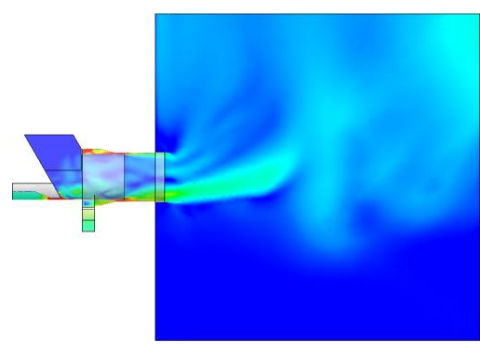

Fechado

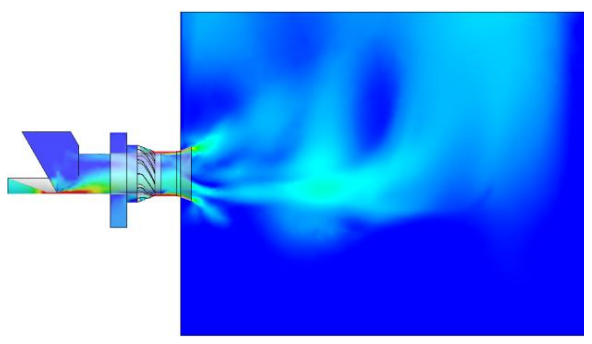

Aberto

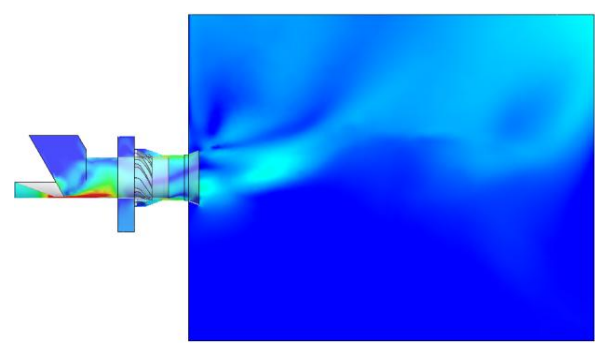

Figura 4: Perfil de vertical iso-simétrico velocidades das simulações de combustão heterogênea de bagaço.

É possível observar que, como esperado, o arranjo aberto apresenta maior penetração e um comportamento com velocidades maiores na direção axial em relação aos demais casos. A configuração fechada do espargidor novo apresenta maior grau de mistura em relação aos demais e o 

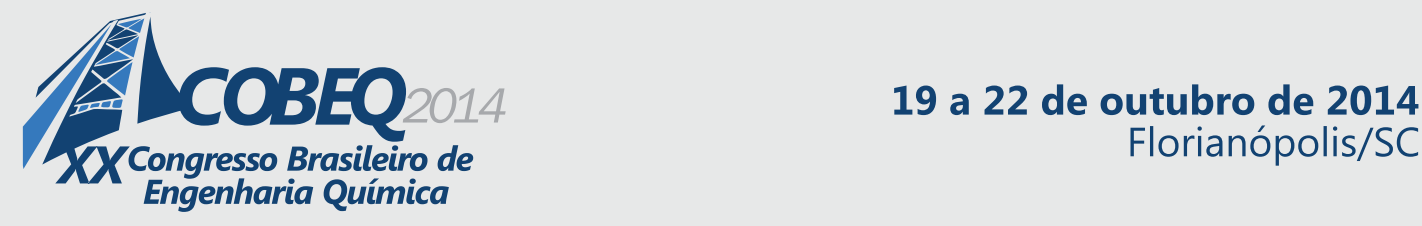

Florianópolis/SC

escoamento promovido pelo modelo atual tem um comportamento intermediário em relação às duas configurações novas.

Como esperado, as maiores partículas tem a tendência de penetrarem mais no interior da fornalha e sofrem menos influência do escoamento. Por outro lado, as menores partículas sofrem mais influência do escoamento e apresentam trajetórias mais abertas. A Figura 5 apresenta as trajetórias de 90 partículas de bagaço em função da fração mássica de carbonizado (char) produzida após a etapa de volatilização do bagaço.
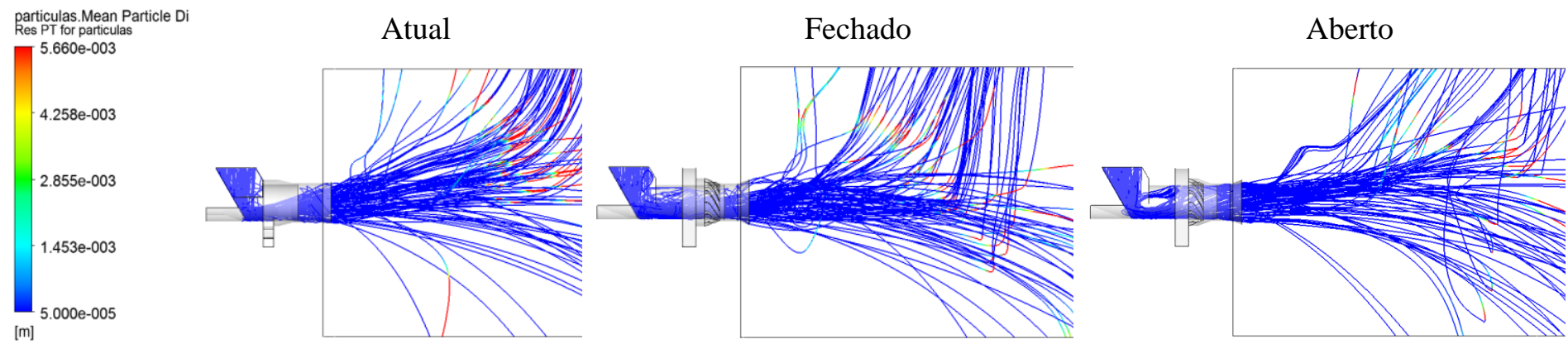

Figura 5: Trajetórias de 90 partículas representadas em função da fração mássica de carbonizado.

É possível observar que, tanto o espargidor atual quanto o novo sob o arranjo fechado, apresentam uma espécie de frente de produção de carbonizado, sugerindo tendência a maior controle da região de volatilização do bagaço. Esse comportamento não é observado no arranjo aberto em que a presença de carbonizado se encontra mais distante da injeção do bagaço. Como a volatilização é uma das etapas dominantes na combustão heterogênea e a formação de carbonizado é muito útil na sua identificação, é esperado que o perfil de temperaturas seja coerente com o comportamento observado para a fração mássica de carbonizado e o perfil de velocidades. A Figura 6 apresenta a renderização volumétrica de temperatura nas vistas frontal e lateral.

Coerente com as renderizações de velocidade e as frações mássicas de carbonizado obtidas, o comportamento térmico não apenas apresenta maiores temperaturas a uma região mais afastada do espargidor para o arranho aberto do modelo novo, como também sugere que o arranjo fechado e o espargidor antigo promovam maior rotação e mistura no farfield. Os resultados obtidos para as frações mássicas de $\mathrm{O}_{2}, \mathrm{CO}, \mathrm{H}_{2} \mathrm{O}$ e $\mathrm{CO}_{2}$ sugerem que suas concentrações seguem o comportamento do perfil térmico, evidenciando que, apesar do modelo de combustão ser governado pela escala de tempo turbulenta, a temperatura e as espécies químicas estão fortemente ligadas. Essa observação reforça a ideia de que a volatilização tem influência dominante sobre a queima heterogênea. Sendo assim, é possível constatar que, comparando os resultados das Figuras 4 a 6, a configuração e geometria do espargidor novo possuem a maior capacidade de controle sobre a queima do bagaço. 

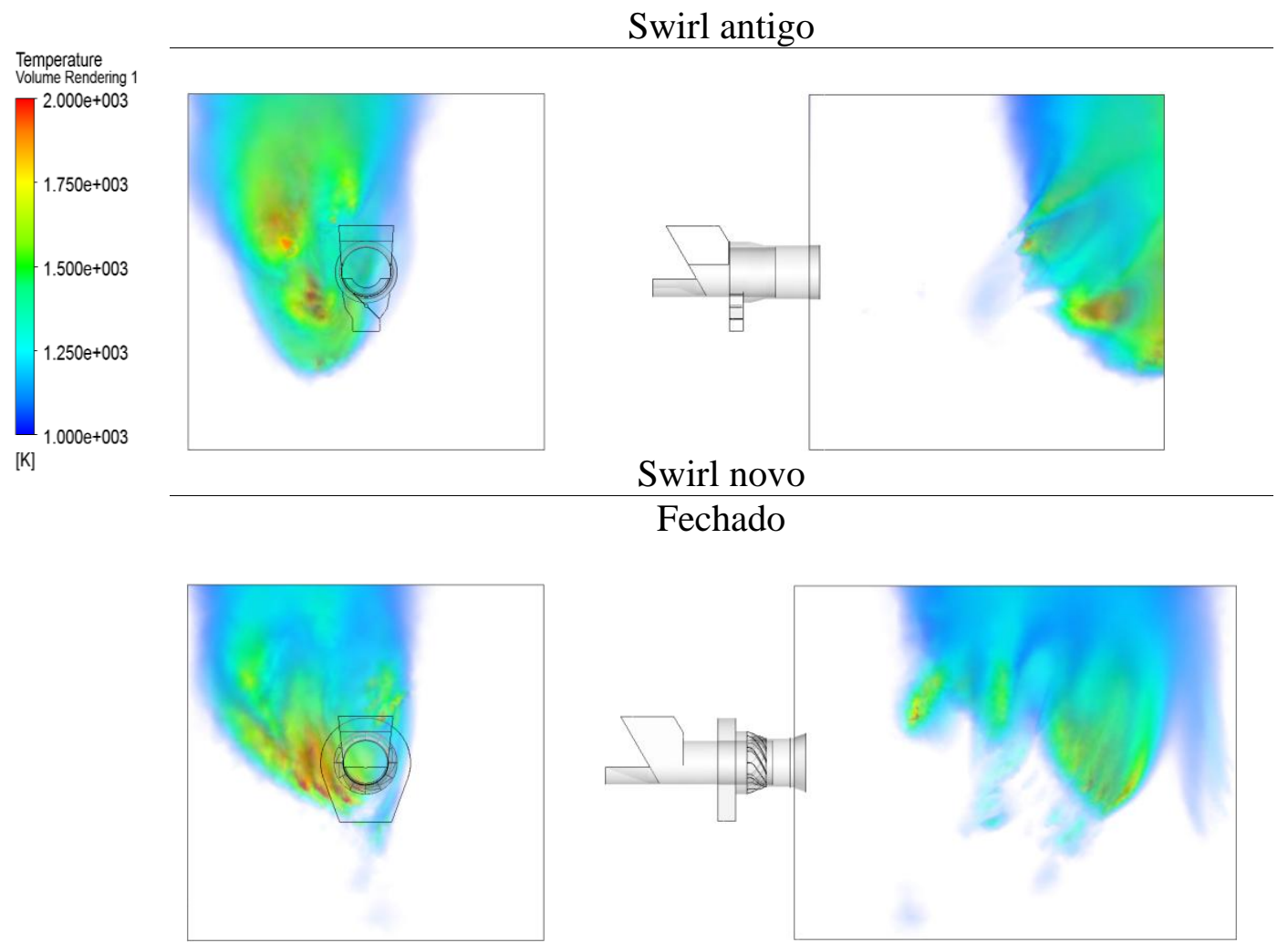

Aberto
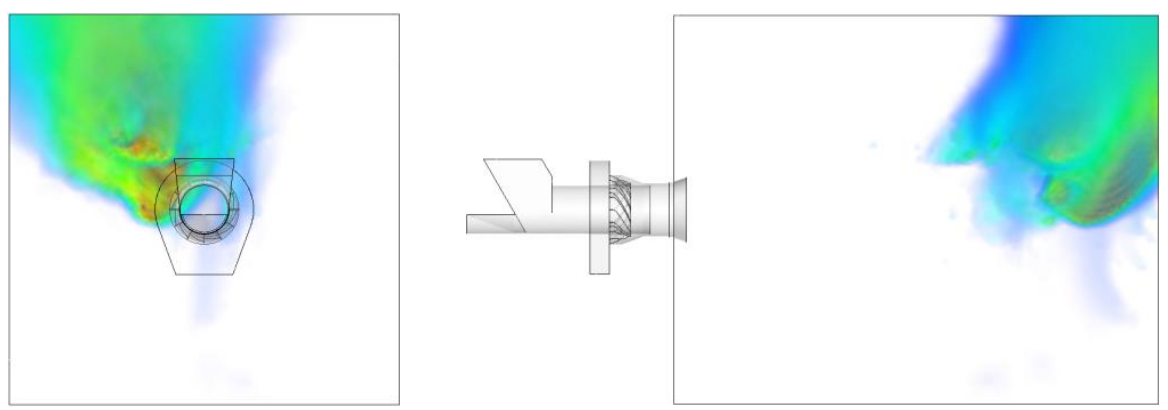

Figura 6: Renderizações volumétricas para temperatura das simulações de combustão heterogênea de bagaço (visões perpendicular ao plano do observador disposto à esquerda e lateral à direita). 


\section{CONCLUSÕES}

O desenvolvimento de um modelo CFD se mostrou útil e interessante para simular e avaliar o projeto de um novo espargidor de bagaço baseado em comparações com o design atual. Os resultados obtidos para as simulações se mostraram coerentes com o comportamento esperado de acordo com os projetos e operação esperada dos espargidores instalados em caldeiras. Mesmo que o modelo de combustão EDM para a fase gasosa seja governado pela turbulência do escoamento, o perfil térmico e os perfis das espécies químicas se mostraram mais dependentes entre si, sugerindo que a combustão heterogênea do bagaço seja mais influenciada pela volatilização do que pelo escoamento. De acordo com as comparações dos resultados para as geometrias testadas, é possível afirmar que o novo design proposto para espargidor em caldeiras permite maior controle da combustão do bagaço se comparado com o equipamento atual cuja operação pode ser considerada um regime intermediário entre as operações das configurações aberta e fechada do espargidor novo.

\section{AGRADECIMENTOS}

Autores agradecem a FAPESP (São Paulo) pelos recursos do Projeto 2010/50389-3 "Aplicação da Fluidodinâmica Computacional a uma Caldeira de Bagaço; e pelo auxílio viagem para participação no presente CoBEQ 2014.

\section{REFERÊNCIAS}

Dixon, T. F.; Mann, A. P.; Plaza, F.; Gilfillan, W. N.; Development of advanced technology for biomass combustion - CFD as an essential tool. Fuel, vol. 84, pp. 1303-1311, 2005.

Ferreira, D.J.O.; Sosa-Arnao, J. H.; Moreira, B.C.; Rangel, L.P.; Park, S.W. Impacto da radiação na modelagem de uma caldeira de bagaço de cana-de-açúcar. COBEQ 2014 - XX Congresso Brasileiro de Engenharia Química. Florianópolis, Brasil. 19-22 de Outubro de 2014.

Modest, M. F.; Radiative Heat Transfer - Second Edition. Academic Press, 2003.

Sosa-Arnao, J. H.; Caldeiras aquatubulares de bagaço - Estudo do sistema de recuperação de energia. Tese de doutorado, UNICAMP, Campinas, SP, 2008

Turns, S. R. An introduction to combustion: concepts and applications. McGraw-Hill, New York, 2000.

Versteeg, H. K.; Malalasekera, W.; An introduction to computational fluid dynamics - the finite volume method. Second edition. England, Pearson Education Limited, 2007. 